\title{
Quantum impurity in the bulk of a topological insulator
}

\author{
Hai-Feng Lü, ${ }^{1,2}$ Hai-Zhou Lu, ${ }^{1}$ Shun-Qing Shen, ${ }^{1}$ and Tai-Kai Ng ${ }^{3}$ \\ ${ }^{1}$ Department of Physics, The University of Hong Kong, Pokfulam Road, Hong Kong, China \\ ${ }^{2}$ Department of Applied Physics, University of Electronic Science and Technology of China, Chengdu 610054, China \\ ${ }^{3}$ Department of Physics, Hong Kong University of Science and Technology, Clear Water Bay Road, Hong Kong, China
}

(Received 20 September 2012; revised manuscript received 13 March 2013; published 15 May 2013)

\begin{abstract}
We investigate physical properties of an Anderson impurity embedded in the bulk of a topological insulator. The slave-boson mean-field approximation is used to account for the strong electron correlation at the impurity. Different from the results of a quantum impurity on the surface of a topological insulator, we find for the band-inverted case that a Kondo resonant peak and in-gap bound states can be produced simultaneously. However, only one of them appears for the normal case. It is shown that the mixed-valence regime is much broader in the band-inverted case, while it shrinks to a very narrow regime in the normal case. Furthermore, a self-screening of the Kondo effect may appear when the interaction between the bound-state spin and impurity spin is taken into account.
\end{abstract}

DOI: 10.1103/PhysRevB.87.195122

PACS number(s): 71.27.+a, 73.20.Hb, 75.20.Hr

\section{INTRODUCTION}

A topological insulator (TI) is insulating in the bulk, but hosts conducting edge or surface states near the system boundary. It has attracted attention in the community of condensed matter physics due to its potential application in spintronics and quantum computation. ${ }^{1-3}$ A class of materials such as $\mathrm{Bi}_{2} \mathrm{Se}_{3}$ and $\mathrm{Bi}_{2} \mathrm{Te}_{3}$ has been found to possess surface states which form a single Dirac cone. ${ }^{4-6}$ Suppression of backscattering inside the Dirac cone guarantees that the Dirac dispersion remains essentially unperturbed for weak perturbation that preserves time-reversal symmetry. ${ }^{7,8}$ So far, the effects of impurity scattering on the surface of TIs have been investigated extensively. ${ }^{9-22}$ In the presence of classical spins which break time-reversal symmetry, it was predicted that the impurity could open up a local gap and suppress the local density of states. ${ }^{9,10}$ The suppression of backscattering around nonmagnetic impurities on surfaces with strong spin-orbit coupling has been confirmed by scanning tunneling miscroscope experiments. ${ }^{11-14}$ However, strong nonmagnetic scattering, such as that from electrostatic potentials, may disrupt the Dirac cone and create low-energy impurity resonances. ${ }^{15}$ For a quantum impurity on the surface of a TI, the Hamiltonian for the impurity can be mapped to the conventional pseudogap Anderson model. The impurity is fully screened at low temperatures when the Fermi level is located away from the Dirac point. ${ }^{16,17}$

Although there are theoretical and experimental studies on the quasiparticle states around an impurity, most of them focus on the impurity on the surface of the TI. Essentially, the topological nature of TIs is determined by the electronic structure of the bulk bands instead of the surface states. On the other hand, the TI samples available nowadays are always poorly insulating in the bulk, owing to a large amount of vacancies and defects. ${ }^{23-27}$ For these reasons, it is important to study how the quasiparticle states are affected when vacancies or impurities are localized in the bulk of the system. It has been shown that classical spins ${ }^{18}$ or vacancies ${ }^{19,20}$ localized in the bulk of TIs could result in the coexistence of in-gap bound states and boundary states. For a quantum impurity, the quantum fluctuations of its internal degree of freedom play an important role, making it significantly differ from classical impurities. ${ }^{28}$ However, it remains unknown how a quantum impurity in the bulk of a TI affects the electronic states.

The study of quantum impurities in TIs is also related to the problems of impurities in unconventional density waves,${ }^{28-31}$ gapped systems, ${ }^{32,33}$ or spin-orbit-coupled systems. ${ }^{34-36}$ For instance, in a gapped system, the Kondo effect breaks down when the energy gap exceeds a critical value. ${ }^{32}$ In the presence of Rashba spin-orbit interaction, a parity-breaking Dzyaloshinsky-Moriya term could be induced, resulting in a possible change of the Kondo temperature. ${ }^{34-36}$ Since there are strong spin-orbit couplings and unconventional gaps in the TIs, it becomes interesting to investigate the differences between the Kondo effects in conventional insulators and TIs.

In this paper, we investigate the effects of a quantum impurity embedded in a TI with the help of the slave-boson mean-field approach. We show that in-gap bound states and Kondo effect could coexist in TIs, while only one of them appears for conventional insulators. If the bound states are singly occupied, the Kondo resonance could be screened by the exchange interaction between the impurity spin and the spin of the impurity-induced bound states, leading to a self-screened Kondo effect. The paper is organized as follows. In Sec. II we introduce a model Hamiltonian of an impurity in a TI and the slave-boson mean-field approach. In Sec. III we discuss the Kondo effect and the formation of the in-gap bound states in both band-inverted and normal cases. In Sec. IV, we show the self-screening of the Kondo effect. Finally, a summary is presented in Sec. V.

\section{MODEL HAMILTONIAN AND SLAVE-BOSON APPROACH}

\section{A. Model}

The effective model to describe the bulk states of TIs with an impurity is written as

$$
H=H_{0}+H_{d}+H_{t}
$$


The part for the bulk electrons of the TI is given by the modified Dirac model ${ }^{37-39}$

$$
H_{0}=\Psi_{k}^{\dagger}\left[\hbar v_{F} \vec{k} \cdot \vec{\alpha}+\left(m v_{F}^{2}-B \hbar^{2} k^{2}\right) \beta\right] \Psi_{k}
$$

with $\alpha_{i}=\sigma_{x} \otimes \sigma_{i}$ and $\beta=\sigma_{z} \otimes \sigma_{0}$, where $\sigma_{i}(i=x, y, z)$ are the Pauli matrices and $\sigma_{0}$ is the $2 \times 2$ unit matrix. $k_{i}=-i \partial_{i}$ $(i=x, y, z)$ is the momentum operator, $k^{2}=k_{x}^{2}+k_{y}^{2}+k_{z}^{2}$, and $v_{F}$ and $m$ have the dimensions of speed and mass, respectively. Different from the surface Hamiltonian, a quadratic correction in momentum $-B \hbar^{2} k^{2}$ and a gap term $m v_{F}^{2}$ are introduced in the bulk Hamiltonian. The sign of $m B$ determines whether the system is topologically trivial or not: it is nontrivial for $m B>0$ (i.e., the band-inverted case), and trivial for $m B<0$ (i.e., the normal case). ${ }^{37-39}$ The energy spectra have a finite energy gap. Here the basis vectors are chosen as

$$
\Psi_{k}^{\dagger}=\left(a_{k \uparrow}^{\dagger}, a_{k \downarrow}^{\dagger}, b_{k \uparrow}^{\dagger}, b_{k \downarrow}^{\dagger}\right)
$$

where $a_{k \sigma}^{\dagger}$ and $b_{k \sigma}^{\dagger}$ are creation operators of electrons with spin $\sigma$ on two different orbits. In this representation, we can rewrite the total Hamiltonian in the second-quantized form

$$
\begin{aligned}
H_{0}= & \sum_{k \sigma}\left(m v_{F}^{2}-B \hbar^{2} k^{2}\right)\left(a_{k \sigma}^{\dagger} a_{k \sigma}-b_{k \sigma}^{\dagger} b_{k \sigma}\right) \\
& +\hbar v_{F} \sum_{k}\left[k_{z}\left(a_{k \uparrow}^{\dagger} b_{k \uparrow}-a_{k \downarrow}^{\dagger} b_{k \downarrow}\right)\right. \\
& \left.+\left(k_{x}-i k_{y}\right)\left(a_{k \uparrow}^{\dagger} b_{k \downarrow}+b_{k \uparrow}^{\dagger} a_{k \downarrow}\right)+\text { H.c. }\right] .
\end{aligned}
$$

The Hamiltonian that describes the Anderson impurity is

$$
H_{d}=\epsilon_{d}\left(c_{d \uparrow}^{\dagger} c_{d \uparrow}+c_{d \downarrow}^{\dagger} c_{d \downarrow}\right)+U c_{d \uparrow}^{\dagger} c_{d \uparrow} c_{d \downarrow}^{\dagger} c_{d \downarrow},
$$

where $\epsilon_{d}$ is the impurity energy level and $U$ is the on-site Coulomb interaction. The coupling Hamiltonian between the impurity and electrons in TI has the form

$$
H_{t}=\sum_{k \sigma}\left(V_{a k} a_{k \sigma}^{\dagger} c_{d \sigma}+V_{b k} b_{k \sigma}^{\dagger} c_{d \sigma}+\text { H.c. }\right),
$$

where $V_{a(b) k}$ represents the overlap or hybridization matrix element between the magnetic impurity and conduction electrons in two bands.

\section{B. Slave-boson mean field}

Here we consider a strong on-site Coulomb interaction on the impurity, i.e., $U \rightarrow \infty$. In this limit, no double occupancy on the impurity is allowed. We introduce the auxiliary fields

$$
c_{d \sigma}^{\dagger}=d_{\sigma}^{\dagger} b, \quad c_{d \sigma}=b^{\dagger} d_{\sigma},
$$

where the boson operator $b^{\dagger}$ creates an empty state and the fermion operator $d_{\sigma}^{\dagger}$ creates a singly occupied state on the impurity. These two fields obey the local constraint $b^{\dagger} b+\sum_{\sigma} d^{\dagger} d=1 .{ }^{40}$ In the mean-field approximation, both the annihilation and creation boson operators $b$ and $b^{\dagger}$ are replaced by a complex number $b_{0}$ and its complex conjugate $b_{0}^{*}$, and the local constraint is realized by introducing a Lagrangian multiplier $\lambda_{0}$. Substituting the auxiliary fields in the original Hamiltonians (3) and (4), one can get

$$
H_{d}=\tilde{\epsilon}_{d}\left(d_{\uparrow}^{\dagger} d_{\uparrow}+d_{\downarrow}^{\dagger} d_{\downarrow}\right)+\lambda_{0}\left(b_{0}^{2}-1\right)
$$

and

$$
H_{t}=\sum_{k \sigma}\left(\tilde{V}_{a k} a_{k \sigma}^{\dagger} d_{\sigma}+\tilde{V}_{b k} b_{k \sigma}^{\dagger} d_{\sigma}+\text { H.c. }\right)
$$

with the renormalized parameters $\tilde{\epsilon}_{d}=\epsilon_{d}+\lambda_{0}$ and $\tilde{V}_{a(b) k}=$ $b_{0} V_{a(b) k}$. The slave-boson mean-field approximation was first introduced to describe the low-energy physics of the conventional Anderson impurity model in the mixedvalence regime. ${ }^{41}$ This method may produce the low-energy physics in unconventional density waves (e.g., $d$-wave superconductors, ${ }^{42}$ graphene electron systems, ${ }^{43}$ etc.).

\section{Green's functions}

Utilizing the method of the equation of motion for the impurity electron, one finally obtains the retarded Green's function of the impurity electron,

$$
\left\langle\left\langle d_{\sigma} \mid d_{\sigma}^{\dagger}\right\rangle\right\rangle=\frac{\omega-\tilde{\epsilon}_{d}-\Sigma_{0}(\omega)+\sigma \Sigma_{z}(\omega)}{\left[\omega-\tilde{\epsilon}_{d}-\Sigma_{0}(\omega)\right]^{2}-\sum_{i}\left[\Sigma_{i}(\omega)\right]^{2}},
$$

where the self-energy functions are defined as

$$
\begin{aligned}
& \Sigma_{0}(\omega)=\sum_{k} \frac{\left(\omega+A_{k}\right) \tilde{V}_{a k}^{2}+\left(\omega-A_{k}\right) \tilde{V}_{b k}^{2}}{\omega^{2}-\hbar^{2} v_{F}^{2} k^{2}-A_{k}^{2}}, \\
& \Sigma_{i}(\omega)=\sum_{k} \frac{2 \hbar v_{F} k_{i} \tilde{V}_{a k} \tilde{V}_{b k}}{\omega^{2}-\hbar^{2} v_{F}^{2} k^{2}-A_{k}^{2}},
\end{aligned}
$$

$(i=x, y, z)$, and $A_{k}=m v_{F}^{2}-B \hbar^{2} k^{2}$.

It is assumed that the hybridization strength $V_{a(b) k}$ does not depend on momentum, $V_{a(b) k}=V_{a(b)}$. In the absence of an external magnetic field, the interaction self-energy $\Sigma_{i}(\omega)=0$ and the electronic Green's function can be simplified as

$$
\left\langle\left\langle d_{\sigma} \mid d_{\sigma}^{\dagger}\right\rangle\right\rangle=\frac{1}{\omega-\tilde{\epsilon}_{d}-\Sigma_{0}(\omega)},
$$

which has the same form as those of the conventional Anderson impurity model. Additionally, we consider the case that the impurity is symmetrically coupled with two orbits, i.e., $V_{0}=$ $V_{a}=V_{b}$, and the self-energy $\Sigma_{0}(\omega)$ in $d$ dimensions $(d=2,3)$ is thus simplified as

$$
\begin{aligned}
\Sigma_{0}(\omega) & =\omega \sum_{k} \frac{2 \tilde{V}_{0}^{2}}{\omega^{2}-\hbar^{2} v_{F}^{2} k^{2}-A_{k}^{2}} \\
& =\frac{d \omega \tilde{V}_{0}^{2} N_{0}}{k_{F}^{d}} \int_{0}^{\infty} d k \frac{k^{d-1}}{\omega^{2}-\hbar^{2} v_{F}^{2} k^{2}-A_{k}^{2}},
\end{aligned}
$$

where $N_{0}$ is the number of lattice sites and $k_{F}$ is the Fermi wave vector for the bulk of a TI.

\section{Normal and band-inverted regimes}

From the poles of self-energy function $\Sigma_{0}(\omega)$, one obtains the energy spectrum of the bulk,

$$
\xi(k)= \pm \sqrt{\hbar^{2} v_{F}^{2} k^{2}+\left(m v_{F}^{2}-B \hbar^{2} k^{2}\right)^{2}} .
$$

The energy spectrum shows a complex dependence on the energy gap and momentum. Such energy spectra can be mapped onto many important cases of impurity problems. Near the band edges, the model reduces to the Anderson problem 


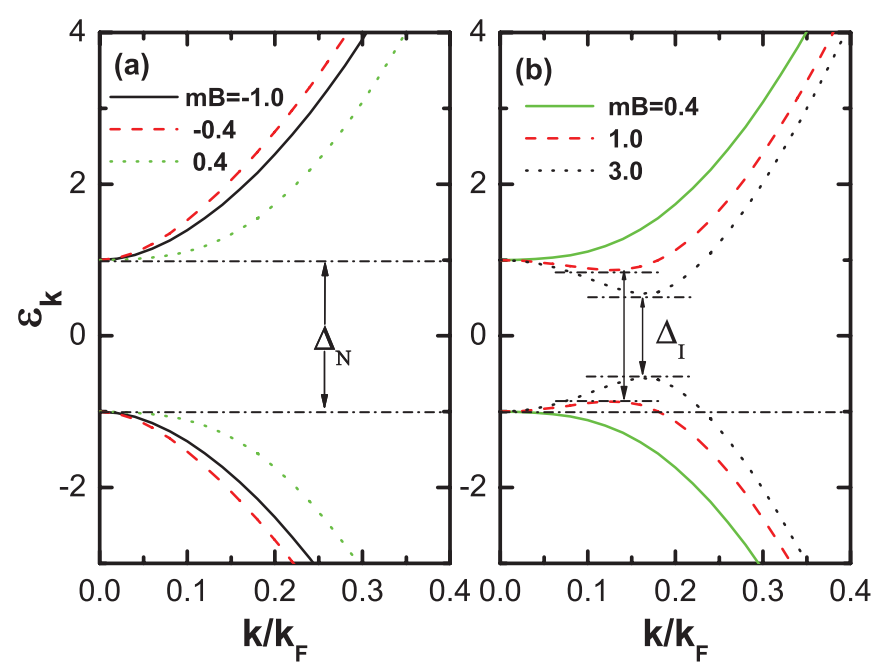

FIG. 1. (Color online) The energy spectrum $\epsilon_{k}$ for different $m B$ values: (a) $m B=-1.0,-0.4,0.4$; (b) $m B=0.4,1.0,3.0$. Here $m v_{F}^{2}$ is taken as the energy unit. For $m B<1 / 2$, the energy gap is located at $k=0$ and equals $\Delta_{N} \equiv 2|m| v_{F}^{2}$. For $m B>1 / 2$, the energy gap is located at a finite $k$ and equals $\Delta_{I} \equiv \frac{\sqrt{4 m B-1}}{|B|} v_{F}^{2}$.

in normal insulators or semiconductors. ${ }^{32,33}$ The quantum impurity model in graphene or surface states of the TIs can be recovered by setting $m=0$ and $B=0 .{ }^{16,17,22,43}$

In Fig. 1 we present the energy spectra as a function of $k$ for different values of $m B$. It can be seen from the energy spectrum that, for $m B<1 / 2$, the band edges are located at $k=0$ and the energy gap is

$$
\Delta_{N} \equiv 2|m| v_{F}^{2}
$$

as in normal insulators. However, for the case of $m B>1 / 2$, the band edges appear at a finite $k$ with a gap

$$
\Delta_{I} \equiv \frac{\sqrt{4 m B-1}}{|B|} v_{F}^{2} .
$$

For convenience, one defines

$$
g_{0}(\epsilon)=\frac{d N_{0} \tilde{V}_{0}^{2} \epsilon\left[\sqrt{\epsilon^{2}-\Delta_{I}^{2} / 4}-\frac{1-2 m B}{2|B|} v_{F}^{2}\right]^{\frac{d-2}{2}}}{4\left(|B| \hbar^{2} k_{F}^{2}\right)^{\frac{d}{2}} \sqrt{\epsilon^{2}-\Delta_{I}^{2} / 4}}
$$

when $\epsilon>\Delta_{N} / 2$, and

$$
g_{1}(\epsilon)=\frac{d N_{0} \tilde{V}_{0}^{2} \epsilon\left[\sqrt{\epsilon^{2}-\Delta_{I}^{2} / 4}+\frac{2 m B-1}{2|B|} v_{F}^{2}\right]^{\frac{d-2}{2}}}{4\left(|B| \hbar^{2} k_{F}^{2}\right)^{\frac{d}{2}} \sqrt{\epsilon^{2}-\Delta_{I}^{2} / 4}}
$$

when both $\Delta_{I} / 2<\epsilon<\Delta_{N} / 2$ and $m B>1 / 2$ are satisfied.

For the case of $m B<1 / 2$, one gets

$$
\Sigma_{0}(\omega)=\int_{\frac{\Delta_{N}}{2}}^{\infty} d \xi g_{0}(\xi)\left[\frac{1}{\omega-\xi}+\frac{1}{\omega+\xi}\right] .
$$

After analytical continuation, $\Sigma_{0}(\omega)=\operatorname{Re} \Sigma_{0}(\omega)+\operatorname{Im} \Sigma_{0}(\omega)$ with

$$
\operatorname{Re} \Sigma_{0}(\omega)=\mathrm{P} \int_{\frac{\Delta_{N}}{2}}^{\infty} d \xi g_{0}(\xi)\left[\frac{1}{\omega-\xi}+\frac{1}{\omega+\xi}\right]
$$

and

$$
\operatorname{Im} \Sigma_{0}(\omega)=-g_{0}(\omega) \Theta\left(|\omega|-\frac{\Delta_{N}}{2}\right)
$$

with $\mathrm{P}$ denoting the principal value.

For the case of $m B>1 / 2$, the real and imaginary parts of the self-energy are given by

$$
\begin{aligned}
\operatorname{Re} & \Sigma_{0}(\omega) \\
= & \mathrm{P}\left[\int_{\frac{\Delta_{I}}{2}}^{\frac{\Delta_{N}}{2}} d \xi g_{2}(\xi)+\int_{\frac{\Delta_{N}}{2}}^{\infty} d \xi g_{0}(\xi)\right]\left(\frac{1}{\omega-\xi}+\frac{1}{\omega+\xi}\right)
\end{aligned}
$$

and

$$
\begin{aligned}
\operatorname{Im} \Sigma_{0}(\omega)= & -\left[g_{2}(\omega) \Theta\left(|\omega|-\frac{\Delta_{I}}{2}\right) \Theta\left(\frac{\Delta_{N}}{2}-|\omega|\right)\right. \\
& \left.+g_{0}(\omega) \Theta\left(|\omega|-\frac{\Delta_{N}}{2}\right)\right],
\end{aligned}
$$

where $g_{2}(\omega)=g_{0}(\omega)+g_{1}(\omega)$. Because $\operatorname{Im} \Sigma_{0}$ is proportional to the density of states of the bulk electrons, the density of states is discontinuous at the point $\Delta_{N} / 2$. For a relatively large $B$ and small $m$, the energy gap reduces to a value much smaller than $\Delta_{N}$.

The free energy $F$ of the system is given by the partition function

$$
F=-\frac{1}{\beta} \ln Z=-\frac{1}{\beta} \int_{-\infty}^{\infty} \ln \left(1+e^{-\beta(\omega-\mu)}\right) \bar{\rho}(\omega) d \omega,
$$

where $\bar{\rho}(\omega)=\Sigma_{k} \delta\left(\omega-\epsilon_{k}\right), \epsilon_{k}$ are the one-electron energies of the system, $\mu$ is the chemical potential of the TI, and $\beta=$ $1 / k_{B} T$ is the system temperature. $\bar{\rho}(\omega)$ can be calculated from the poles of the one-electron Green's function and is given by

$$
\bar{\rho}(\omega)=\frac{\operatorname{Im}}{\pi} \sum_{k} \frac{4 \omega}{\omega^{2}-\hbar^{2} v_{F}^{2} k^{2}-A_{k}^{2}}+2 \frac{\operatorname{Im}}{\pi} \frac{\partial}{\partial \omega} \ln \left\langle\left\langle d_{\sigma} \mid d_{\sigma}^{\dagger}\right\rangle\right\rangle .
$$

Minimizing the free energy of the system with respect to $\lambda_{0}$ and $b_{0}$, one obtains a set of self-consistent equations

$$
\begin{array}{r}
2 \int_{-\infty}^{\infty} d \omega f(\omega) \rho_{d}(\omega)+b_{0}^{2}-1=0, \\
2 \int_{-\infty}^{\infty} d \omega f(\omega)\left(\omega-\tilde{\epsilon}_{d}\right) \rho_{d}(\omega)+\lambda_{0} b_{0}^{2}=0,
\end{array}
$$

where $f(\omega)=\left[1+e^{(\omega-\mu) / k_{B} T}\right]^{-1}$ is the Fermi distribution function and the density of states $\rho_{d}(\omega)$ of the impurity is given by

$$
\rho_{d}(\omega)=-\frac{1}{\pi} \frac{\operatorname{Im} \Sigma_{0}(\omega)}{\left[\omega-\tilde{\epsilon}_{d}-\operatorname{Re} \Sigma_{0}(\omega)\right]^{2}+\operatorname{Im} \Sigma_{0}(\omega)^{2}} .
$$

In the calculation, it is limited to zero temperature, i.e., $k_{B} T=0 . \Delta_{N} / 2$ is taken as the energy unit and $\Gamma_{0}=$ $\pi \rho_{0} V_{0}^{2}$ represents the hybridization strength between the impurity and the bulk electrons, where $\rho_{0}=N_{0} / 2 D$ is the density of states per spin at the chemical potential and $D=$ $\sqrt{\left(\hbar v_{F} k_{F}\right)^{2}+\left(m v_{F}^{2}-B \hbar^{2} k_{F}^{2}\right)^{2}}$ is a cutoff of the band width. In the following, we show the results of quantum impurity in three-dimensional TIs for $\Gamma_{0}=0.5$ and $D=30.0$. 


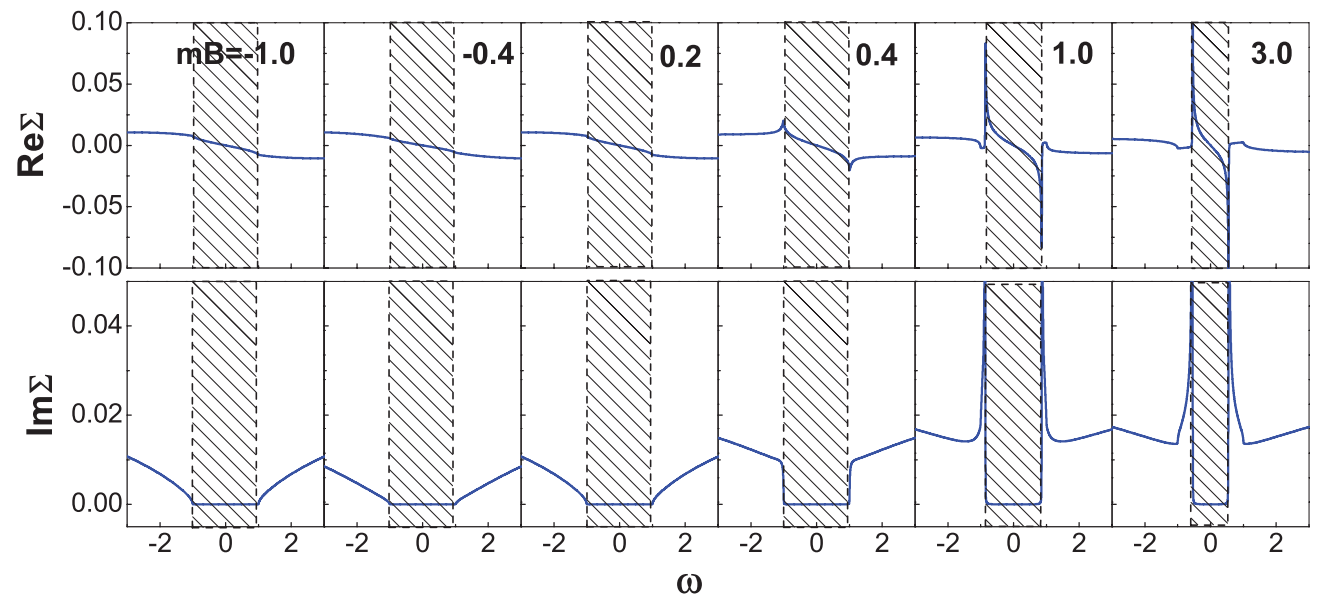

FIG. 2. (Color online) The real and imaginary parts of the self-energy $\Sigma_{0}(\omega)$ for different $m B$ values. The shaded part represents the region of energy gap.

\section{IN-GAP BOUND STATES AND THE KONDO EFFECT}

\section{A. Self-energy}

First, we discuss the self-energy $\Sigma_{0}(\omega)$, which depends on the energy spectrum of the bulk bands. In the bulk of the TI, the strong spin-orbit coupling couples the conduction and valence bands, leading to the nonparabolic energy spectrum. The self-energy $\Sigma_{0}(\omega)$ reveals not only the poles of the Green's function, but also the inhomogeneous density of states due to the impurity-induced states.

Figure 2 shows the real and imaginary parts of $\Sigma_{0}(\omega)$ versus $\omega$ for different values of $m B$. Previous studies based on the Chern number and $Z_{2}$ invariant indicate that the band-inverted case with $m B>0$ is topologically nontrivial, while the normal case with $m B<0$ is topologically trivial. ${ }^{37-39}$ It is shown that in Fig. 2 both the real and imaginary parts of $\Sigma_{0}(\omega)$ are quite different for opposite signs of $m B$. The real part $\operatorname{Re} \Sigma_{0}(\omega)$ determines the level positions of both the Kondo resonance and the bound states, according to the solutions to the equation

$$
\omega=\tilde{\epsilon}_{d}+\operatorname{Re} \Sigma_{0}(\omega)
$$

For $m B>1 / 2, \operatorname{Re} \Sigma_{0}(\omega)$ diverges rapidly near the edges of the gap, thus there always exist bound states within the gap. When the impurity level $\left|\epsilon_{d}\right|$ is much larger than $\Delta_{I} / 2$, the bound states are very close to the bottom of the conduction bands for $\epsilon_{d} \gg \Delta_{I} / 2$ or the top of the valence band for $\epsilon_{d} \ll-\Delta_{I} / 2$, which is similar to the case of $s$-wave superconductors. ${ }^{44}$ More importantly, Eq. (17) has an extra solution in the band region, corresponding to the coexisting Kondo resonance. For $m B<$ 0 , there is only one solution to Eq. (17), so the Kondo effect and the bound states do not appear simultaneously.

The imaginary part $\operatorname{Im} \Sigma_{0}(\omega)$ is proportional to the density of states of bulk electrons. For $m B>1 / 2$, the energy gap is $\Delta_{I}$, thus $\operatorname{Im} \Sigma_{0}(\omega)=0$ in the region of $\omega \in\left[-\Delta_{I} / 2, \Delta_{I} / 2\right]$. At the band edges $\pm \Delta_{I} / 2$, Im $\Sigma_{0}(\omega)$ shows divergences. In the region $|\omega| \in\left[\Delta_{I} / 2, \Delta_{N} / 2\right]$, the divergences drop rapidly with the increasing $|\omega|$. For $|\omega|>\Delta_{N} / 2, \operatorname{Im} \Sigma_{0}(\omega)$ begins to increase as a function of $\sqrt{\omega}$. For $0<m B<1 / 2$, the divergences at the band edges $\pm \Delta_{N} / 2$ disappear gradually with decreasing $m B$. For $m B<0$, $\operatorname{Im~} \Sigma_{0}(\omega)$ at the band edges is always zero and increases as a function of $\sqrt{\omega}$ for $|\omega|>\Delta_{N} / 2$.

\section{B. Density of states of the impurity}

From the self-energy, the Kondo resonance and the bound states have been discussed qualitatively. The density of states $\rho_{d}(\omega)$ of the impurity for the cases that the chemical potential $\mu$ lies in the gap and in the valence bands are presented in Figs. 3 and 4, respectively. It is demonstrated that in both cases the Kondo resonance and bound states coexist for $m B>0$, while only one of them appears for $m B<0$. For $m B>0$, the bound states are very close to the band edges when $\tilde{\epsilon}_{d}$ is far away from the energy gap. In the previous studies, ${ }^{31-33}$ it has been argued that, when the energy gap exceeds a critical value $\left(\Delta / T_{K}=2.0\right.$ predicted by slave-boson mean-field theory ${ }^{32}$ and $T_{K}$ is the Kondo temperature), the Kondo effect no longer appears in insulators with an energy-independent density of states. However, for a complex dispersion relation, the density of states is strongly energy resolved and quite different near the band edges for the band-inverted and normal cases. In the band-inverted case, $\rho_{d}\left( \pm \Delta_{I} / 2\right)$ equals zero exactly, due to the divergence of $\operatorname{Im} \Sigma_{0}(\omega)$ near the band edges. For $|\omega| \gg \Delta_{I}, \rho_{d}(\omega)$ also approaches zero. Therefore, no matter how $\mu$ lies in the gap or in the bands, there are always two low-energy resonance peaks lying near the band edges for $m B>1 / 2$, as shown in the upper panels of Figs. 3 and 4 . When the chemical potential $\mu$ lies in the valence band, the low-energy resonance peak becomes narrow and its position moves to $\mu$ with decreasing $\epsilon_{d}$. Near the critical point $\epsilon_{d c}$, the resonance peak becomes very sharp and close to the chemical potential.

In Fig. 5 we present the positions of the in-gap bound states and low-energy Kondo resonance as functions of the impurity level $\epsilon_{d}$ in the normal and band-inverted cases. The bound states show quite different behaviors in the two cases. For the normal case, the position of the bound states is almost linear in $\epsilon_{d}$. The in-gap bound states start from the point $\epsilon_{d c}=-\Delta_{N} / 2$, corresponding to the top of the valence bands. At the bottom of the conduction bands, the bound states enter the conduction bands continuously, and 


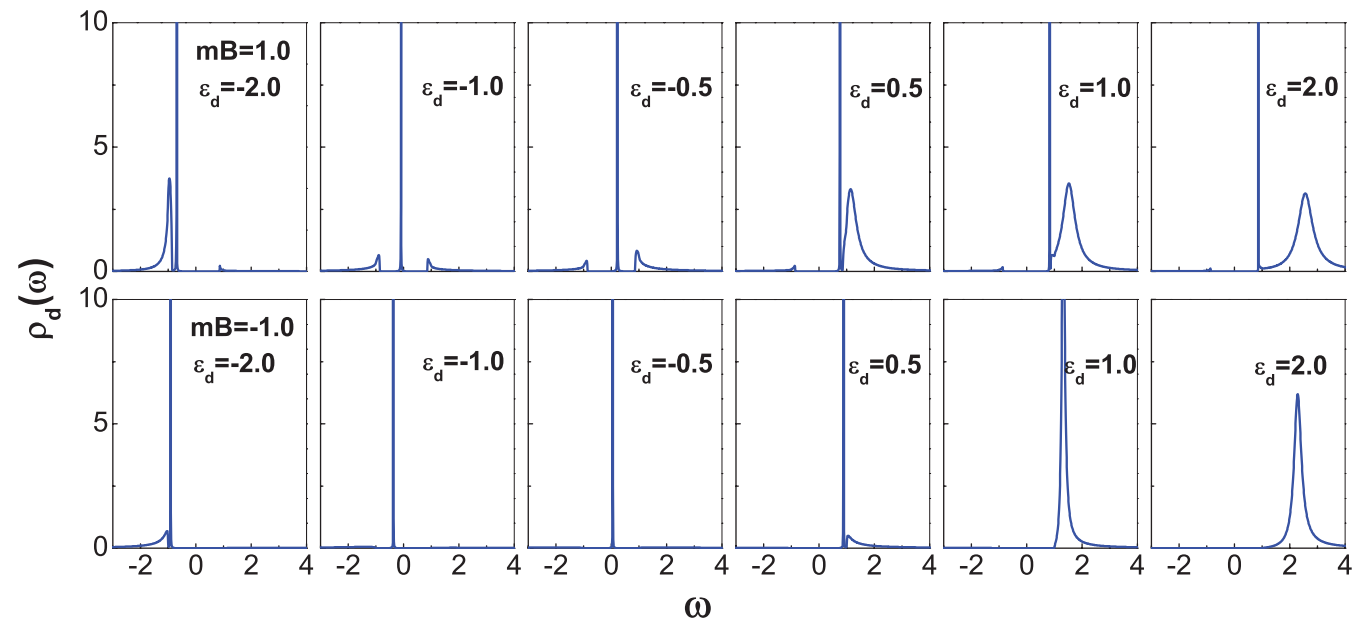

FIG. 3. (Color online) The density of states of the impurity electron is shown for different impurity levels $\epsilon_{d}=-2.0,-1.0,-0.5,0.5$, 1.0 , and 2.0 (from left to right) in (a) topological nontrivial case $m B=1.0$ and (b) topological trivial case $m B=-1.0$, where the chemical potential $\mu=0.0$ lies in the gap.

then the low-energy Kondo resonance peak appears. However, for the band-inverted case, the bound states in the gap and the Kondo resonance can form simultaneously for the impurity level $\epsilon_{d}>\epsilon_{d c}$. In this case, the positions of these two states do not connect at any point. For an $\epsilon_{d}$ far away from the chemical potential, the in-gap bound states are very close to the band edges. Thus they are vulnerable to small perturbation or thermal fluctuation, and may merge into the conduction bands easily.

Above we demonstrate that the presence of the in-gap bound states is determined by the topological nature of the TI. For real TI materials such as $\mathrm{Bi}_{2} \mathrm{Se}_{3}$ and $\mathrm{Bi}_{2} \mathrm{Te}_{3}$, the system parameters from first-principles calculations ${ }^{4}$ are $\left(m v_{F}^{2}, \hbar v_{F}, B\right)=\left(0.28 \mathrm{eV}, 3.2 \mathrm{eV} \AA, 33 \mathrm{eV} \AA^{2}\right)$ and $(0.30 \mathrm{eV}$, $2.9 \mathrm{eV} \AA, 57 \mathrm{eV} \AA^{2}$ ), respectively. Correspondingly, $m B \sim 0.9$ for $\mathrm{Bi}_{2} \mathrm{Se}_{3}$ and 2.0 for $\mathrm{Bi}_{2} \mathrm{Te}_{3}$. Therefore, it is expected that the coexistence of in-gap bound state and Kondo effect could be observed in these two materials.

\section{Broadened mixed-valence regime for the band-inverted case}

The mean field $b_{0}^{2}$ introduced in Sec. II B gives the probability that the impurity is empty. Figure 6 presents the dependence of $b_{0}^{2}$ on the impurity energy level $\epsilon_{d}$ for different values of $m B \cdot b_{0}^{2}=0$ when the impurity level $\epsilon_{d}$ is much lower than the chemical potential, which means that the impurity is singly occupied. In this case, the charge fluctuation between the impurity and the bulk bands is suppressed. When $\epsilon_{d}$ exceeds a threshold value, $b_{0}^{2}$ begins to increase from 0 and saturates at 1 when $\epsilon_{d} \gg \mu$. The mixed-valance regime is defined as where $b_{0}^{2}$ changes from 0 to 1 . Figure 6 presents $b^{2}$ when the chemical potential lies in the gap and in the valence bands. As shown in Fig. 6, the mixed-valence regime is broader for the band-inverted case with $m B>0$. For the normal case with $m B<0, b_{0}^{2}$ increases more rapidly, and the mixed-valence regime shrinks into a very narrow regime. This kind of narrow mixed-valence regime has also been found in unconventional density waves and $d$-wave superconductors. ${ }^{28,31}$ For $m B<0$,

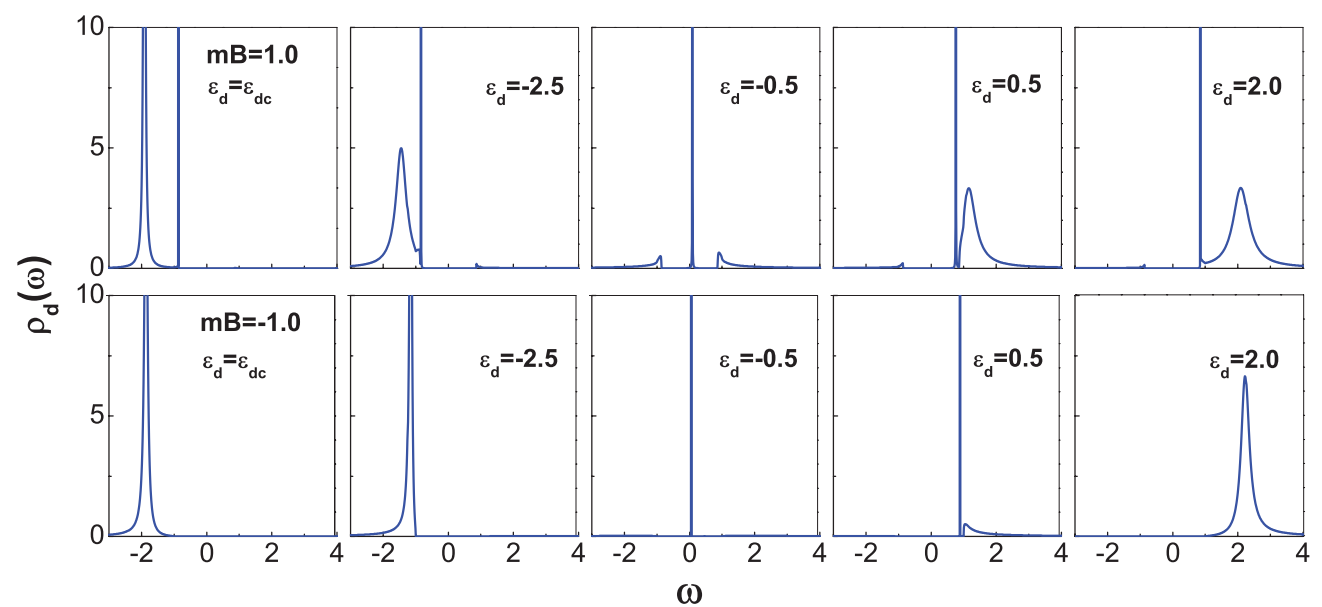

FIG. 4. (Color online) The density of states of the impurity electron is shown for different impurity levels $\epsilon_{d}=\epsilon_{d c}\left(\epsilon_{d c}\right.$ is the critical point at which $\left.\tilde{\epsilon}_{d}=\mu\right),-2.5,-0.5,0.5$, and 2.0 (from left to right) in (a) topological nontrivial case $m B=1.0$ and (b) topological trivial case $m B=-1.0$, where the chemical potential $\mu=-2.0$ lies in the valence band. 

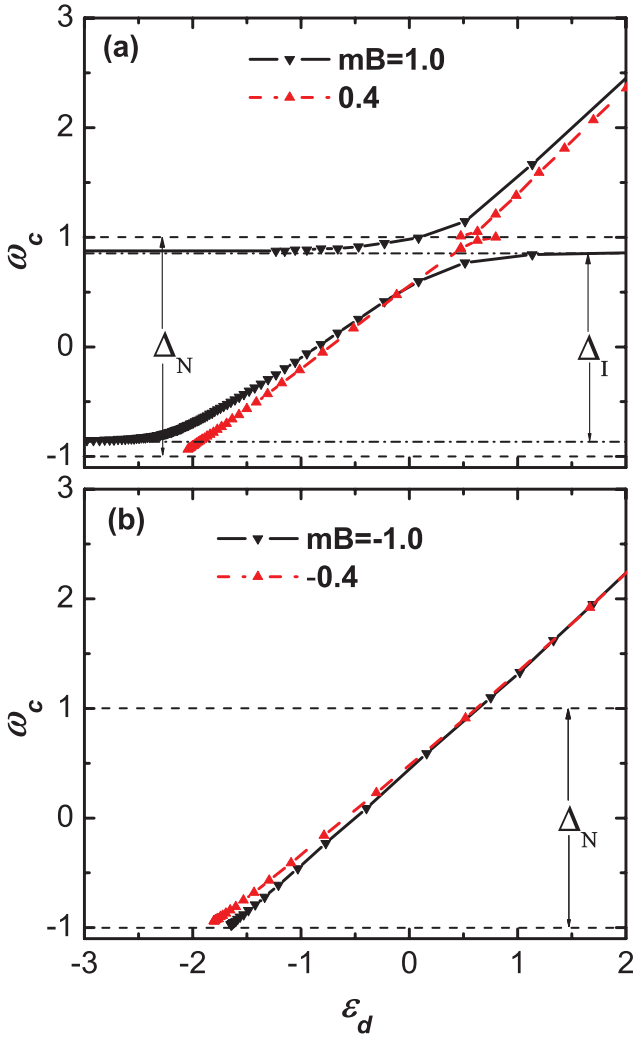

FIG. 5. (Color online) The positions of the bound states and lowenergy Kondo resonance peak as functions of impurity level $\epsilon_{d}$ in (a) the topological nontrivial case $m B=1.0,0.4$ and (b) the topological trivial case $m B=-1.0,-0.4$. The chemical potential is taken as $\mu=0.0$.

the density of states of bulk electrons vanishes at the band edges. The reduction of the density of states at the band edges implies that the Kondo resonance peak is narrower and $b_{0}^{2}$ decreases to zero faster than those in the conventional case. From the self-consistent equations, near the critical point, the critical value of $\epsilon_{d}$ is determined by

$$
\epsilon_{d c}=2 \int_{-D}^{\mu} d \omega \frac{g(\omega)}{\omega-\tilde{\epsilon}_{d}},
$$

where $g(\omega)$ is proportional to the density of states of the background electrons. In the band-inverted case, $g(\omega)$ diverges near the band edges, which results in a large integral value as well as large $\left|\epsilon_{d c}\right|$. In contrast, $g(\omega)$ reduces to zero near the band edges in the normal case. In this case, $\left|\epsilon_{d c}\right|$ is smaller and the mixed-valence regime becomes narrower.

\section{In two dimensions}

The preceding sections present the numerical results in three dimensions. In this section, we briefly discuss the behaviors of the quantum impurity in the bulk of two-dimensional TIs. In practice, the two-dimensional case is more accessible by the scanning tunneling miscroscope. ${ }^{14}$ At the mean-field level, the difference between two and three dimensions arises from the self-energy $\Sigma_{0}(\omega)$, which in two dimensions can be
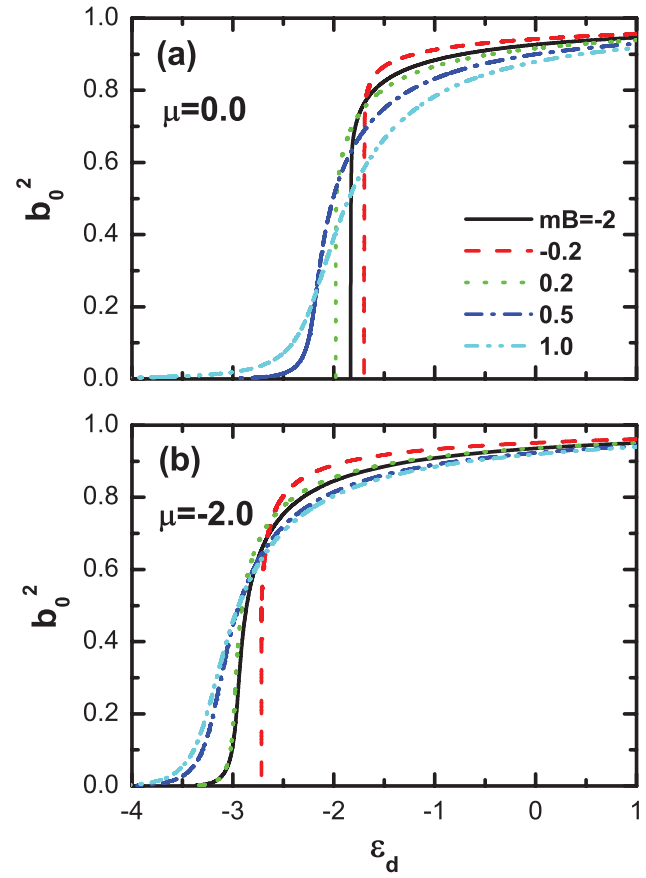

FIG. 6. (Color online) The dependence of the order parameter $b_{0}^{2}$ on the impurity energy level $\epsilon_{d}$ for (a) the chemical potential in the gap $\mu=0.0$ and (b) in the valence band $\mu=-2.0$. Here $m B=-2.0$ and -0.2 represent the topological nontrivial case and $m B=0.2,0.5$, and 1.0 correspond the topological trivial case.

expressed as

$$
\Sigma_{0}(\omega)=\frac{2 \omega \tilde{V}_{0}^{2} N_{0}}{k_{F}^{d}} \int_{0}^{\infty} d k \frac{k}{\omega^{2}-\hbar^{2} v_{F}^{2} k^{2}-A_{k}^{2}} .
$$

A detailed discussion about the properties of $\Sigma_{0}(\omega)$ in two dimensions is presented in Ref. 45 , in which a $\delta$-impurity scattering is considered. It can be deduced that, at the point $\omega=\Delta_{N} / 2$, the self-energy $\Sigma_{0}(\omega)$ is finite in three dimensions, while in two dimensions $\Sigma_{0}(\omega)$ has logarithmic divergence to $-(+) \infty$ at $\omega \rightarrow+(-)|m|$ for TIs and has logarithmic divergence to $+(-) \infty$ at $\omega \rightarrow-(+)|m|$ for normal insulators. ${ }^{45}$ This means that, when the Anderson impurity couples only to one band, the topological phase transition can be seen from the position of impurity bound states as the system changes from normal insulator to TI. Besides, the physical properties of the quantum impurity are similar qualitatively in two and three dimensions; e.g., the mixed-valence regime in both cases is much broader for $m B>0$ than the case of $m B<0$.

\section{SELF-SCREENING OF THE KONDO EFFECT}

We have shown that the quantum impurity may induce in-gap bound states in the band-inverted case, leading to the coexistence of the Kondo resonance and the bound states. This indicates that the Kondo resonance and bound state originate from two different mechanisms. The singly-occupied quantum impurity behaves like a single spin, and gives the Kondo resonance when the system is in the Kondo regime. The bound state, on the other hand, is induced when the system is topologically nontrivial. Even a potential scattering ${ }^{18}$ or a vacancy ${ }^{19}$ could produce the in-gap bound state in the bulk 
(a)
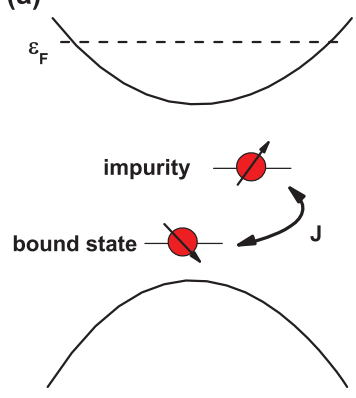

topologically nontrivial case (b)

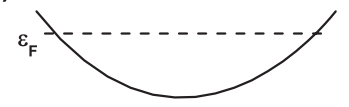

impurity

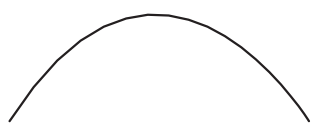

topologically trivial case

FIG. 7. (Color online) Illustration of the Kondo effect for (a) topological nontrivial case and (b) trivial case.

of TIs. Therefore, the in-gap bound state could be considered as a degree of freedom, while its energy is determined by the impurity. When the chemical potential of the TI lies in the conduction bands, both the bound states and the impurity level are occupied, and each of them behaves like a single localized spin (as illustrated in Fig. 7). It is quite natural to expect the exchange interaction between two quantum spins, which is usually due to second-order virtual hopping or the Ruderman-Kittel-Kasuya-Yosida (RKKY) mechanism mediated by itinerant electrons. If the interaction is antiferromagnetic, the two spins form a singlet, which will compete with the many-body singlet formed by the impurity spin and conduction electrons, quenching their Kondo effect. Different from the intensively studied two-impurity Kondo problem, ${ }^{46,47}$ here the quenched Kondo effect is induced by the spin of the bound states rendered by the impurity itself. Therefore, we refer to this effect as the self-screening of the Kondo effect.

\section{A. Exchange interaction between impurity spin and impurity-induced bound-state spin}

The self-screened Kondo effect can be illustrated by the model Hamiltonian as follows:

$$
\begin{aligned}
H= & H_{0}+\sum_{\sigma} \epsilon_{d} d_{\sigma}^{\dagger} d_{\sigma}+\sum_{\sigma} \epsilon_{f} f_{\sigma}^{\dagger} f_{\sigma}+J \mathbf{S}_{d} \cdot \mathbf{S}_{f} \\
& +\sum_{k \sigma}\left(V_{d} b_{d} a_{k \sigma}^{\dagger} d_{\sigma}+V_{d} b_{d} b_{k \sigma}^{\dagger} d_{\sigma}+\text { H.c. }\right) \\
& +\sum_{k \sigma}\left(V_{f} b_{f} a_{k \sigma}^{\dagger} f_{\sigma}+V_{f} b_{f} b_{k \sigma}^{\dagger} f_{\sigma}+\text { H.c. }\right) \\
& +\lambda_{d}\left(\sum_{\sigma} d_{\sigma}^{\dagger} d_{\sigma}+b_{d}^{2}-1\right)+\lambda_{f}\left(\sum_{\sigma} f_{\sigma}^{\dagger} f_{\sigma}+b_{f}^{2}-1\right)
\end{aligned}
$$

where $\epsilon_{f}$ is the impurity-induced bound-state level and $H_{0}$ is the Hamiltonian for the bulk of the TI [Eq. (2)]. The term $J \mathbf{S}_{d}$. $\mathbf{S}_{f}$ describes the exchange interaction between the impurity spin and bound-state spin. It is noted that here the quantum impurity and its induced in-gap bound state are considered as two degrees of freedom.

The above effective Hamiltonian describes the low-energy behavior of the Anderson impurity in the nontrivial TI phase of the system. It can be derived from a renormalization-group approach where the high-energy degree of the system is

integrated out systematically. The derivation of the effective coupling between the bound state and Kondo resonance requires a technique beyond the slave-boson mean-field theory, which was presented recently. ${ }^{48}$ Using a weak coupling renormalization group analysis, it has been shown that the exchange interaction $J$ between the $d$ and induced $f$ spins may be renormalized dynamically to either positive or negative values. In the regime where charge fluctuations in both $d$ and $f$ states are quenched, the system is in the selfscreened Kondo regime for $J>0$ and in the $\mathrm{SO}(3)$ Kondo regime for $J<0,{ }^{48}$ respectively. Here we introduce the exchange interaction $J$ and perform the slave-boson approach to describe the Kondo physics for small charge fluctuations in the regime of $J>0$.

\section{B. Order parameter for the exchange interaction}

Similar to the treatment in Sec. II B, two slave-boson operators $b_{d}$ and $b_{f}$ are introduced to replace $c_{d(f) \sigma}$ by $b_{d(f)}^{\dagger} d(f)_{\sigma}$ in the large- $U$ limit. The spin exchange term $J \mathbf{S}_{d}$. $\mathbf{S}_{f}=J \sum_{\sigma, \sigma^{\prime}} d_{\sigma}^{\dagger} d_{\sigma^{\prime}} f_{\sigma^{\prime}}^{\dagger} f_{\sigma}$ can be decoupled by introducing a valence-bond field $\Delta_{0}=-\sum_{\sigma}\left\langle d_{\sigma}^{\dagger} f_{\sigma}\right\rangle$. In the mean-field approximation $^{47}$

$$
J \mathbf{S}_{d} \cdot \mathbf{S}_{f} \rightarrow J \Delta_{0} \sum_{\sigma}\left(d_{\sigma}^{\dagger} f_{\sigma}+f_{\sigma}^{\dagger} d_{\sigma}\right)+J \Delta_{0}^{2},
$$

the Hamiltonian becomes quadratic in the fermion operators. The problem is still far from trivial as $b_{d}, b_{f}, \lambda_{d}, \lambda_{f}, \Delta_{0}$, and $\epsilon_{f}$ need to be determined self-consistently. Different from the ordinary Kondo problem, here the bound-state energy $\epsilon_{f}$ also enters the self-consistent equations. By minimizing the ground-state energy, one obtains a set of nonlinear selfconsistent equations,

$$
\begin{aligned}
& \sum_{\sigma}\left\langle d_{\sigma}^{\dagger} d_{\sigma}\right\rangle+b_{d}^{2}-1=0, \\
& \sum_{\sigma}\left\langle f_{\sigma}^{\dagger} f_{\sigma}\right\rangle+b_{f}^{2}-1=0, \\
& \sum_{\sigma}\left(\left\langle d_{\sigma}^{\dagger} f_{\sigma}\right\rangle+\left\langle f_{\sigma}^{\dagger} d_{\sigma}\right\rangle\right)+2 \Delta_{0}=0, \\
& \sum_{k, \sigma}\left(V_{d}\left\langle a_{k \sigma}^{\dagger} d_{\sigma}\right\rangle+V_{d}\left\langle b_{k \sigma}^{\dagger} d_{\sigma}\right\rangle+\text { H.c. }\right)+2 b_{d} \lambda_{d}=0, \\
& \sum_{k, \sigma}\left(V_{f}\left\langle a_{k \sigma}^{\dagger} f_{\sigma}\right\rangle+V_{f}\left\langle b_{k \sigma}^{\dagger} f_{\sigma}\right\rangle+\text { H.c. }\right)+2 b_{f} \lambda_{f}=0 .
\end{aligned}
$$

To simplify the calculation, we assume that the bound states are always singly occupied $\left(b_{f}=0\right)$ and decoupled from the conduction electrons. In this case, the in-gap bound states act like a single spin. Correspondingly, the constraints for the bound states becomes $\lambda_{f}\left(\sum_{\sigma}\left\langle f_{\sigma}^{\dagger} f_{\sigma}\right\rangle-1\right)$ and $\lambda_{f}$ is contained in the related Green's functions.

\section{Green's functions}

Performing the equation-of-motion procedure, we can obtain the Green's functions for the impurity and the bound 
states,

$$
\begin{aligned}
& \left\langle\left\langle d_{\sigma} \mid d_{\sigma}^{\dagger}\right\rangle\right\rangle=\frac{1}{\omega-\tilde{\epsilon}_{d}-\frac{J^{2} \Delta_{0}^{2}}{\omega-\tilde{\epsilon}_{f}}-\Sigma_{0}(\omega)}, \\
& \left\langle\left\langle f_{\sigma} \mid f_{\sigma}^{\dagger}\right\rangle\right\rangle=\frac{1}{\omega-\tilde{\epsilon}_{f}-\frac{J^{2} \Delta_{0}^{2}}{\omega-\tilde{\epsilon}_{d}-\Sigma_{0}(\omega)}},
\end{aligned}
$$

where $\quad \tilde{\epsilon}_{d}=\epsilon_{d}+\lambda_{d}, \quad \tilde{\epsilon}_{f}=\epsilon_{f}+\lambda_{f}, \quad$ and $\quad \Sigma_{0}(\omega)=$ $\omega \sum_{k} \frac{2 \tilde{V}_{0}^{2}}{\omega^{2}-\hbar^{2} v_{F}^{2} k^{2}-A_{k}^{2}}$.

In the limit $\Delta_{0} \rightarrow 0$, the impurity and the bound states are decoupled and the results reduce to those when $J=0$. When the spin exchange interaction exceeds a critical $J_{c}$, a nonzero order parameter $\Delta_{0}$ appears and the Kondo peak near the chemical potential is expected to split. From the Green's functions $\left\langle\left\langle d_{\sigma} \mid d_{\sigma}^{\dagger}\right\rangle\right\rangle$, one obtains the self-consistent equation for the bound states,

$$
\epsilon_{f}-\tilde{\epsilon}_{d}-\frac{J^{2} \Delta_{0}^{2}}{\epsilon_{f}-\tilde{\epsilon}_{f}}-\Sigma_{0}\left(\epsilon_{f}\right)=0 .
$$

Defining $\alpha(\omega)=\left[\omega-\tilde{\epsilon}_{d}-\operatorname{Re} \Sigma_{0}(\omega)\right]\left(\omega-\tilde{\epsilon}_{f}\right)-J^{2} \Delta_{0}^{2}$ and $\beta(\omega)=\operatorname{Im} \Sigma_{0}(\omega)\left(\omega-\tilde{\epsilon}_{f}\right)$, the self-consistent equations are derived as

$$
\begin{aligned}
-\frac{2}{\pi} \int_{-\infty}^{\mu} d \omega \frac{\left(\omega-\tilde{\epsilon}_{f}\right) \beta(\omega)}{\alpha(\omega)^{2}+\beta(\omega)^{2}}+b_{d}^{2}-1 & =0, \\
-\frac{2}{\pi} \int_{-\infty}^{\mu} d \omega \frac{J^{2} \Delta_{0}^{2} \operatorname{Im} \Sigma_{0}(\omega)}{\alpha(\omega)^{2}+\beta(\omega)^{2}} & =1, \\
-\frac{2}{\pi} \int_{-\infty}^{\mu} d \omega \frac{J \Delta_{0} \beta(\omega)}{\alpha(\omega)^{2}+\beta(\omega)^{2}}+\Delta_{0} & =0, \\
\frac{2}{\pi} \int_{-\infty}^{\mu} d \omega \frac{\left[\left(\omega-\tilde{\epsilon}_{d}\right)\left(\omega-\tilde{\epsilon}_{f}\right)-J^{2} \Delta_{0}^{2}\right] \beta(\omega)}{\alpha(\omega)^{2}+\beta(\omega)^{2}} & =\lambda_{d} b_{d}^{2}, \\
\alpha\left(\epsilon_{f}\right) & =0 .
\end{aligned}
$$

This set of equations can be solved numerically.

\section{Self-screened Kondo effect}

Figure 8 presents the effects of bound-state spin on the Kondo effect for different exchange interaction strength $J$. The chemical potential of the bulk of the TI is tuned into the conduction bands, so there is a Kondo peak near the chemical potential and the bound states are occupied by a single electron. When $J$ exceeds a critical value $J_{c}$, the order parameter $\Delta_{0}$ begins to increase from zero, then quickly to 1 with the increase of $J$. Figure 8(b) shows the density of states of the impurity as a function of energy for $J=0.0,0.04$, and 0.08 . With the increase of $J$, the resonance peak splits. The splitting of the Kondo peak increases with $J$. As a result, the density of states near $\tilde{\epsilon}_{d}$ reduces to a very small value, corresponding to the self-screening of the Kondo resonance.

It should be noted that the strength of exchange interaction between the impurity and the in-gap bound state is the key parameter of the predicted self-screened Kondo effect. The exchange strength should be evaluated subtly; for instance, from the first-principles calculation. Our calculation indicates that a small exchange interaction could make the Kondo effect break down.
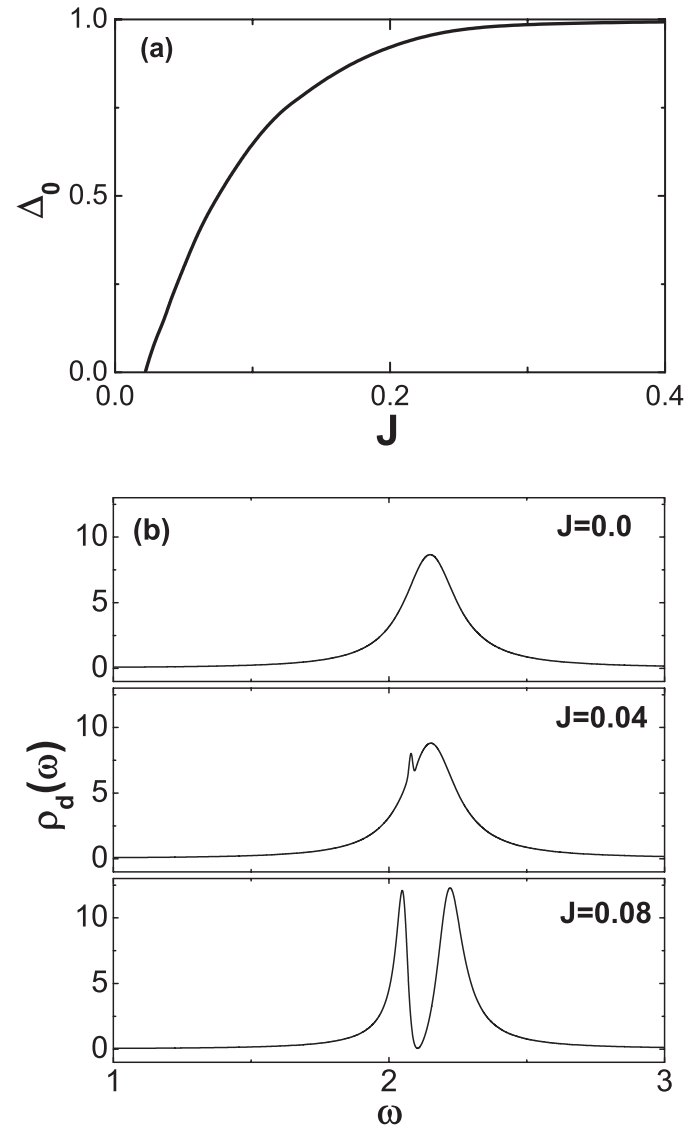

FIG. 8. (a) The order parameter $\Delta_{0}$ as a function of $J$. Parameters: $\epsilon_{d}=1.0, \mu=2.0$, and $m B=1.0$. (b) The density of states of the impurity for $J=0.0,0.04$, and 0.08 .

Above we assumed that the bound states are singlyoccupied in the large- $U$ limit. If the Coulomb repulsion energy on the bound states is finite and the chemical potential is high enough, it is possible that the bound states are occupied by two electrons, and they form a singlet due to the Pauli exclusion principle. In this limit, the bound-state spin is decoupled from the impurity spin, and the Kondo effect originating from the interaction between the impurity spin and conduction electrons can recover.

An impurity- or vacancy-induced in-gap bound state is a special feature of TIs, which is absent in normal insulators. Therefore, the bound state may play an important role when the Kondo physics is considered in TIs. For a strong exchange interaction between impurity and in-gap bound state, the Kondo effect may be broken down when the chemical potential is tuned properly. Actually, several experiments have been performed to investigate the physical properties of magnetic impurity-doped topological materials, such as $\mathrm{Mn}$-doped BiTe. ${ }^{49}$ The self-screening effect is expected to be observed in these systems.

\section{SUMMARY}

In summary, we have studied the Kondo effect and the formation of in-gap bound states induced by an Anderson impurity coupled with the bulk states of topological insulators. 
It is demonstrated that the positions of the Kondo peak and bound states strongly depend on the topological properties, the chemical potential, and other parameters of the system. The behaviors of the resonance level in the bulk of TIs differ from those for simple metals and normal insulators. Due to the divergence of the density of states near the band edges, the mixed-valence regime is much broader in the band-inverted case, while it shrinks to a very narrow regime in the normal case. For the band-inverted case, the in-gap bound states and the Kondo resonance can coexist. However, only one of them exists in the normal insulators. When the impurity energy level is far away from the chemical potential, the in-gap bound states are very close to the band edges and can be considered as merging into the bulk. Furthermore, we show that a self-screening Kondo effect may be induced by taking the interaction between the impurity spin and bound-state spin into account.

Note added: While this manuscript was under review, we became aware of a work ${ }^{50}$ wherein the scattering of dilute magnetic impurities placed on the surface of TIs is investigated.

\section{ACKNOWLEDGMENTS}

This project was supported by the Research Grant Council under Grants No. HKU 7051/10P and No. HKUST3/CRF/09, the Foundation for Innovative Research Groups of the NSFC under Grant No. 61021061, and the NSFC under Grants No. 11004022 and No. 61006081.
${ }^{1}$ M. Z. Hasan and C. L. Kane, Rev. Mod. Phys. 82, 3045 (2010).

${ }^{2}$ X. L. Qi and S. C. Zhang, Rev. Mod. Phys. 83, 1057 (2011).

${ }^{3}$ J. E. Moore, Nature (London) 464, 194 (2010).

${ }^{4}$ H. Zhang, C. X. Liu, X. L. Qi, X. Dai, Z. Fang, and S. C. Zhang, Nat. Phys. 5, 438 (2009).

${ }^{5}$ Y. L. Chen et al., Science 325, 178 (2009).

${ }^{6}$ Y. Xia et al., Nat. Phys. 5, 398 (2009).

${ }^{7}$ C. Wu, B. A. Bernevig, and S. C. Zhang, Phys. Rev. Lett. 96, 106401 (2006).

${ }^{8}$ C. Xu and J. E. Moore, Phys. Rev. B 73, 045322 (2006).

${ }^{9}$ Q. Liu, C. X. Liu, C. Xu, X. L. Qi, and S. C. Zhang, Phys. Rev. Lett. 102, 156603 (2009).

${ }^{10}$ R. R. Biswas and A. V. Balatsky, Phys. Rev. B 81, 233405 (2010).

${ }^{11}$ P. Roushan, J. Seo, C. V. Parker, Y. S. Hor, D. Hsieh, D. Qian, A. Richardella, M. Z. Hasan, R. J. Cava, and A. Yazdani, Nature (London) 460, 1106 (2009).

${ }^{12}$ T. Zhang, P. Cheng, X. Chen, J. F. Jia, X. Ma, K. He, L. Wang, H. Zhang, X. Dai, Z. Fang, X. C. Xie, and Q. K. Xue, Phys. Rev. Lett. 103, 266803 (2009).

${ }^{13}$ Z. Alpichshev, J. G. Analytis, J. H. Chu, I. R. Fisher, Y. L. Chen, Z. X. Shen, A. Fang, and A. Kapitulnik, Phys. Rev. Lett. 104, 016401 (2010).

${ }^{14}$ Z. Alpichshev, R. R. Biswas, A. V. Balatsky, J. G. Analytis, J. H. Chu, I. R. Fisher, and A. Kapitulnik, Phys. Rev. Lett. 108, 206402 (2012).

${ }^{15}$ A. M. Black-Schaffer and A. V. Balatsky, Phys. Rev. B 85, 121103(R) (2012).

${ }^{16}$ R. Žitko, Phys. Rev. B 81, 241414 (2010).

${ }^{17}$ M. T. Tran and K. S. Kim, Phys. Rev. B 82, 155142 (2010).

${ }^{18}$ Q. Liu and T. X. Ma, Phys. Rev. B 80, 115216 (2009).

${ }^{19}$ W. Y. Shan, J. Lu, H. Z. Lu, and S. Q. Shen, Phys. Rev. B 84, 035307 (2011).

${ }^{20}$ J. Lu, W. Y. Shan, H. Z. Lu, and S. Q. Shen, New J. Phys. 13, 103016 (2011).

${ }^{21}$ L. Andrew Wray et al., Nat. Phys. 7, 32 (2010).

${ }^{22}$ X. Y. Feng, W. Q. Chen, J. H. Gao, Q. H. Wang, and F. C. Zhang, Phys. Rev. B 81, 235411 (2010).

${ }^{23}$ J. G. Checkelsky, Y. S. Hor, M. H. Liu, D. X. Qu, R. J. Cava, and N. P. Ong, Phys. Rev. Lett. 103, 246601 (2009).

${ }^{24}$ K. Eto, Z. Ren, A. A. Taskin, K. Segawa, and Y. Ando, Phys. Rev. B 81, 195309 (2010).
${ }^{25}$ Z. Ren, A. A. Taskin, S. Sasaki, K. Segawa, and Y. Ando, Phys. Rev. B 82, 241306(R) (2010).

${ }^{26}$ D. X. Qu, Y. S. Hor, J. Xiong, R. J. Cava, and N. P. Ong, Science 329, 821 (2010).

${ }^{27}$ H. Peng, K. Lai, D. Kong, S. Meister, Y. Chen, X.-L. Qi, S.-C. Zhang, Z.-X. Shen, and Y. Cui, Nat. Mater. 9, 225 (2010).

${ }^{28}$ A. V. Balatsky, I. Vekhter, and J. X. Zhu, Rev. Mod. Phys. 78, 373 (2006).

${ }^{29}$ D. Withoff and E. Fradkin, Phys. Rev. Lett. 64, 1835 (1990).

${ }^{30}$ C. Gonzalez-Buxton and K. Ingersent, Phys. Rev. B 57, 14254 (1998).

${ }^{31}$ B. Dóra, Phys. Rev. B 71, 075107 (2005).

${ }^{32}$ J. Ogura and T. Saso, J. Phys. Soc. Jpn. 62, 4364 (1993).

${ }^{33}$ M. R. Galpin and D. E. Logan, Phys. Rev. B 77, 195108 (2008).

${ }^{34}$ M. Zarea, S. E. Ulloa, and N. Sandler, Phys. Rev. Lett. 108, 046601 (2012).

${ }^{35}$ R. Žitko and J. Bonča, Phys. Rev. B 84, 193411 (2011).

${ }^{36}$ E. Eriksson, A. Ström, G. Sharma, and H. Johannesson, Phys. Rev. B 86, 161103(R) (2012).

${ }^{37}$ H. Z. Lu, W. Y. Shan, W. Yao, Q. Niu, and S. Q. Shen, Phys. Rev. B 81, 115407 (2010).

${ }^{38}$ W. Y. Shan, H. Z. Lu, and S. Q. Shen, New J. Phys. 12, 043048 (2010).

${ }^{39}$ S. Q. Shen, W. Y. Shan, and H. Z. Lu, Spin 1, 33 (2011).

${ }^{40}$ A. C. Hewson, The Kondo Problem to Heavy Fermions (Cambridge University Press, Cambridge, 1993).

${ }^{41}$ P. Coleman, Phys. Rev. B 29, 3035 (1984).

${ }^{42}$ J. X. Zhu and C. S. Ting, Phys. Rev. B 63, 020506 (2000).

${ }^{43}$ Z. G. Zhu and J. Berakdar, Phys. Rev. B 83, 195404 (2011).

${ }^{44}$ K. Machida and F. Shibata, Prog. Theor. Phys. 47, 1817 (1972).

${ }^{45}$ C. Chan and T. K. Ng, Phys. Rev. B 85, 115207 (2012).

${ }^{46}$ B. A. Jones, C. M. Varma, and J. W. Wilkins, Phys. Rev. Lett. 61, 125 (1988).

${ }^{47}$ R. López, R. Aguado, and G. Platero, Phys. Rev. Lett. 89, 136802 (2002).

${ }^{48}$ I. Kuzmenko, Y. Avishai, and T. K. Ng, arXiv:1303.0911.

${ }^{49}$ Y. S. Hor, P. Roushan, H. Beidenkopf, J. Seo, D. Qu, J. G. Checkelsky, L. A. Wray, D. Hsieh, Y. Xia, S. Y. Xu, D. Qian, M. Z. Hasan, N. P. Ong, A. Yazdani, and R. J. Cava, Phys. Rev. B 81, 195203 (2010).

${ }^{50}$ A. K. Mitchell, D. Schuricht, M. Vojta, and L. Fritz, Phys. Rev. B 87, 075430 (2013) 